\title{
Impact of Starch Content on Protein Adsorption Characteristics in Amphiphilic Hybrid Graft Copolymers
}

Arijit Sengupta ${ }^{1}$, Allison R. Linehan ${ }^{1}$, Peter M. lovine ${ }^{1,2}$

${ }^{1}$ Department of Chemistry and Biochemistry, University of San Diego, San Diego, CA 92110

${ }^{2}$ To whom correspondences should be addressed:

Peter M. lovine

Department of Chemistry and Biochemistry

University of San Diego

5998 Alcala Park

San Diego, CA 92110

Phone: (619) 260-4028

Fax: (619) 260-2211

Email: piovine@sandiego.edu 


\section{Abstract}

Amphiphilic hybrid graft copolymers were synthesized using a graft-to methodology and their protein adsorption profiles studied. Three different hydrophilic side chains were studied: hydroxypropylated high amylose starch, maltodextrin, and polyethylene glycol (PEG). In the high amylose starch compositions, there was a pronounced decrease in protein adsorption with increasing polysaccharide content. As the starch content in the graft copolymers increased from $10 \mathrm{wgt} \%$ to $53 \mathrm{wgt} \%$, BSA protein adsorption decreased by $83 \%$ whereas fibrinogen adsorption was reduced by $40 \%$. Comparisons between the starch-containing hybrid polymers and their respective hydrophobic urethanelinked polyesters were also made. Hybrid 53, containing 53 wgt \% starch, showed a $85 \%$ reduction in BSA adsorption and $51 \%$ reduction in fibrinogen relative to their urethane-linked polyester backbone controls. Grafting branched high amylopectin-derived maltodextrin to the synthetic polymer backbones also conferred modest protein resistance to the hydrophobic backbone polymer. Lastly, it was found that a high amylose graft structure provided comparable, if not slightly more effective, protein resistance compared to a similarly constructed PEG-containing amphiphilic copolymer. 


\section{INTRODUCTION}

Addressing the pervasive issue of nonspecific protein adsorption to biomaterial surfaces is an ongoing challenge that impacts multiple scientific disciplines and industries [1-5]. The amount and identity of adsorbed proteins is believed to modulate foreign-body reactions and ultimately contribute to the long-term success or failure of a material. Poly(ethylene glycol) (PEG) is currently the "gold standard" for protein repellent materials [6-9] as it not only endows protein resistance but it is also biocompatible, soluble in a range of solvents, and structurally well defined with tunable molecular weights. There are, however, some limitations of PEG including the lack of chemically addressable backbone functionality and its propensity to auto-oxidize [10-13]; considering these limitations, there is growing interest in identifying suitable PEG alternatives [1418].

Along these lines, glycopolymers show tremendous versatility in antifouling applications. Early work by Mrkisch demonstrated the use of mannitol-terminated self-assembled monolayers (SAMS) to prevent the adsorption of proteins and the attachment of cells [19] Liedberg and co-workers also studied glycosylated SAMS and found that partial methylation of the terminal galactose moiety gave the lowest adsorption profile [20]. More recently, Maynard

and coworkers have investigated trehalose pendant copolymers synthesized by reversible addition-fragmentation chain-transfer polymerization (RAFT) [21], dextran [22], carboxymethyldextrans [23], pullulan [24] and hyaluronic acid [25] have all been used to study protein-glycopolymer surface interactions. 
Starch-based biomaterials are abundant in the literature yet most studies focus on blend characteristics or synthesizing functional starches using graftfrom [26-28] strategies. Recently, we reported a novel synthetic approach to hybrid amphiphilic graft copolymers that feature starch macromolecules as side chain moieties [29]. In our approach, starch macromolecular reagents are grafted-to an azide-containing urethane linked polyester (ULPE-N3) using copper-catalyzed azide-alkyne cycloaddition chemistry (CuAAC) to give amphiphilic copolymers. The density of starch grafts in the final hybrid polymer is controlled by two factors: the number of reactive sites on the synthetic polymer (i.e. pendant azides) and the stoichiometry of the CuAAC reaction.

Here we evaluate the effectiveness of this new class of starch-containing graft copolymers as antifouling coatings. More broadly, we probe whether or not starch can function as a hydrophilic protein resistant component of an amphiphilic copolymer. Quartz crystal microbalance (QCM) was used to quantitate the extent of protein adsorption in spin-coated polymer films. QCM has emerged as a powerful tool to measure protein adsorption, assess the dynamics associated with complex formation on a wide variety of materials including soft polymeric surfaces $[30-32,35]$. A series of amphiphilic graft copolymers were synthesized differing only in their starch content (10 wgt \%, 36 wgt $\%, 53$ wgt \%). We investigated the interaction of two common proteins, bovine serum albumin (BSA) and fibrinogen, with our starch-containing amphiphilic polymer coatings. Importantly, in order to extract meaningful comparative conclusions, the QCM results for the starch-containing polymers 
were benchmarked against appropriate hydrophobic "base polymer" controls that lacked polysaccharide grafts. In addition, we sought to compare our starchcontaining graft copolymers with similarly constructed PEG analogs. Along these lines, we synthesized two PEG-containing graft copolymers using the same backbone polymers as in the starch series. Also, we sought to understand how structural variation in the starch domains (i.e. branching) impacts protein adsorption. In order to accomplish this goal we synthesized maltodextrin graft copolymers (as compared to predominantly linear high amylose starch) and compared their protein adsorption profiles to that of the high amylose starch.

\section{EXPERIMENTAL}

2.1 Materials. Molecular weight modified starch (hydroxypropylated Hylon VII, $\mathrm{M}_{\mathrm{n}}$ $=6500 \mathrm{kDa}$ ) was received from Ingredion Inc. as a gift. 4,4'-Methylenebis(phenyl isocyanate) (MDI), maltodextrin (DE 4-7, rice starch origin), 1,1,1,3,3,3hexafluoro-2-propanol (HFIP), dibutyltin dilaureate, 4-ethynyl aniline, sodium triacetoxyborohydride, polycaprolactone diol $\left(M_{n}=2000 \mathrm{kDa}\right)$, bovine serum albumin (BSA, lyophilized powder), fibrinogen from human plasma (35 - 65\% protein), sodium dodecyl sulfate (SDS), dimethyl sulfoxide, dichloromethane and methanol were purchased from Sigma Aldrich and used as received. Phosphate buffer saline (10x PBS, $\mathrm{pH}=7.4)$ was also purchased from Sigma Aldrich and diluted to $1 \mathrm{x}$ before use. Ethylenediaminetetraacetic acid (EDTA) was received from Fisher and used as received. All common chemicals were analytical grade. Methoxy-terminated PEG (mPEG) alkyne $\left(M_{n}=2000 \mathrm{kDa}\right)$ was purchased from 
Creative PEGWorks and used as received. The chain extender, 11-azidoundecyl 3-hydroxy-2-(hydroxymethyl)-2-methylpropanoate was synthesized according to the procedure reported by Altintas et.al [33]. AT-cut gold coated sensors (fundamental frequency $5 \mathrm{MHz}$ ) for QCM study were purchased from Biolin Scientific and rinsed thoroughly prior to use.

2.2 Synthesis. All graft copolymers (including the PEG-containing polymers) and macromolecular reagents were synthesized using published procedures [29]. As reported previously, the ULPE-N3 backbone polymers used in these studies had $\mathrm{M}_{\mathrm{n}} \sim 66 \mathrm{~K}$ and PDI's of $\sim 2$ [29]. Azide and alkyne densities associated with the ULPE-N3 backbone polymers and starch reagents, respectively, were calculated using our published protocol[29]. Characterization data and particular compound-specific synthetic details for all new compositions can be found in the Supporting Information. Below we present a general procedure that was applied to the synthesis of both starch-containing hybrid polymers and newly synthesized PEG-grafted amphiphilic polymers.

Briefly, the ULPE-N3 and end-functionalized starch were added to a pressure tube and brought into a glove box. In the glove box, the $\mathrm{CuBr} / \mathrm{N}, \mathrm{N}, \mathrm{N}^{\prime}, \mathrm{N}^{\prime}, \mathrm{N}^{\prime \prime}-$ pentamethyldiethylenetriamine (PMDETA) $(\sim 0.021 \mathrm{M}$ in both $\mathrm{CuBr}$ and PMDETA) solution (2X equiv relative to azide content in the ULPE-N3) was added by syringe to the pressure tube, the reaction concentration adjusted to $8-17 \%$ (w/w) using supplemental dimethylformamide (DMF), and the vessel sealed. After removal from the glove box, the reaction was heated at $40{ }^{\circ} \mathrm{C}$ for 
$18 \mathrm{~h}$. The green-grey solution was cooled to room temperature and precipitated into ice-cold methanol (15X volume of solution) containing $0.5 \mathrm{mM}$ EDTA and the resulting precipitate isolated by centrifugation (3400 rpm, 8-10 min). The solid polymer was dissolved in warm dimethyl sulfoxide (DMSO) $\left(60{ }^{\circ} \mathrm{C}, 8 \%(\mathrm{w} / \mathrm{w})\right)$ and reprecipitated into ice-cold methanol containing $0.5 \mathrm{mM}$ EDTA. The hybrid polymer was isolated by centrifugation ( $3400 \mathrm{rpm}, 8-10 \mathrm{~min}$ ) and washed with one portion of $\mathrm{CH}_{2} \mathrm{Cl}_{2}(20 \mathrm{~mL} / \mathrm{g}$ polymer $)$ to remove any unreacted urethanelinked polyester, filtered, and subsequently washed with two portions of warm water $\left(35^{\circ} \mathrm{C}, 15 \mathrm{~mL} / \mathrm{g}\right.$ polymer) to remove any unreacted starch. The final hybrid graft copolymer was dried by lyophilization.

The graft density in the hybrid polymers was calculated using the same method as we have previously reported [29]. Briefly, unique NMR spectroscopic signatures were integrated and normalized for each component of the hybrid polymer: MDI, azide- containing chain extender, polycaprolactone, and starch (the anomeric proton). Using the ${ }^{1} \mathrm{H}$ NMR-derived molar ratios, and knowing the repeat unit molecular weight for each component of the hybrid, the actual weight percent starch was calculated. The theoretical wgt $\%$ starch was calculated using the reaction stoichiometry and the macromolecular loading values. The graft density was determined by comparing the theoretical wgt \% of starch in the hybrid polymer to the actual wgt $\%$ of starch found in the hybrid.

2.3 QCM Protocols: Unused AT-cut gold coated sensors were thoroughly rinsed with deionized (DI) water and soaked in $2 \%$ SDS solution (40 min). The sensors 
were removed, washed with DI water again, and dried under a gentle stream of nitrogen. The dried sensors were treated with ozone (UV/Ozone Procleaner, Bioforce Nanosciences) (10 $\mathrm{min})$, soaked in basic piranha solution $\left(\mathrm{H}_{2} \mathrm{O} / \mathrm{NH}_{3} / \mathrm{H}_{2} \mathrm{O}_{2}: 5 / 1 / 1\right)\left(15 \mathrm{~min}\right.$ at $\left.75^{\circ} \mathrm{C}\right)$, rigorously rinsed with $\mathrm{DI}$ water, and again dried under nitrogen. One final ozone treatment (10 min) was completed before use. Previously coated sensors were cleaned in an analogous manner except that they were first soaked and rinsed in warm DMSO to remove spincoated polymer.

The clean and dried sensors were spin-coated with polysaccharidecontaining hybrid polymers from a uniform dispersion in HFIP $(0.3 \mathrm{mg} / \mathrm{mL})$ at 2500 rpm using Chemat Technology Spin Coater (KW-4A). The PEG-containing hybrid polymers and the ULPE-N3 base polymers (ULPE-N3-133 and ULPE-N3182) were spin coated from a THF solution $(0.3 \mathrm{mg} / \mathrm{mL})$. All coated sensors were annealed at $50^{\circ} \mathrm{C}$ under vacuum for $12 \mathrm{~h}$ prior to use.

The working principle of QCM has been described elsewhere [34]. Briefly, the instrument monitors the change in resonant frequency of AT-cut gold sensor when a mass is adsorbed on to the surface. If the adsorbed layer is thin, rigid, and non-dissipating $(\Delta D \approx 0)$, the mass change can be correlated directly to the frequency change using the Sauerbrey relationship (eq. 1):

$$
\Delta m=-C \frac{\Delta f}{n} \ldots .(\text { eq. } 1)
$$

where, $\Delta f=$ change in resonance frequency 


$$
\begin{aligned}
& \Delta \mathrm{m}=\text { change in mass } \\
& \mathrm{C}=\text { sensitivity factor of the sensor }\left(17.7 \mathrm{ng} \mathrm{cm}^{-2} \mathrm{~s}^{-1}\right) \\
& \mathrm{n}=\text { overtone }
\end{aligned}
$$

If the adsorbed layer is thick and loosely held, there will be a quick decay in the sensor oscillation resulting in high dissipation. In such cases, the viscoelastic property of the adsorbed material is taken in to account and the adsorbed mass is calculated using the Voigt model [35]. All QCM-derived mass data presented herein was calculated using the Sauerbrey equation. The Sauerbrey model was implemented across all data as $\Delta D \approx 0$ for all overtones. The mass data are reported in terms of mean with standard deviations and $\mathrm{t}$ tests performed where appropriate.

Protein solutions were prepared at a concentration of $1 \mathrm{mg} / \mathrm{mL}$ in PBS buffer $(1 \mathrm{x}, \mathrm{pH}=7.4)$. Polymer coated sensors were first monitored in their dry state in order to establish a baseline. PBS buffer was then introduced at a flow rate of $0.15 \mathrm{~mL} / \mathrm{min}$ and the baseline reestablished at ambient temperature. At this point the intake solution was changed to the protein of interest $(1 \mathrm{mg} / \mathrm{mL})$ flowing at a rate of $0.15 \mathrm{~mL} / \mathrm{min}$. After $20-25$ min under flow, equilibrium was reached as indicated by minimal frequency change over several minutes. At this point, the protein solution was discontinued and replaced by fresh PBS at the same flow rate; the purpose of the final PBS wash was to remove any loosely bound protein from the surface of the polymer. The reported adsorbed mass was calculated after reaching equilibrium on the final PBS wash. All measurements 
were done in triplicate and data extracted from the $3^{\text {rd }}$ overtone using the QTool software (QSense).

2.4 Determination of Dry Coating Thickness by QCM. The dry thickness of the coatings was determined by "stitching" (QSense) two real time data sets which included stable equilibration periods ( $\sim 3 \mathrm{~min})$ of the bare gold sensor and that of the hybrid/base polymer coated gold sensor in air. The $3^{\text {rd }}$ harmonic $\left(\Delta F_{3}\right)$ of the resulting data series was plotted using the Sauerbrey equation and the amount of polymer deposited per unit area $\left(\mathrm{ng} / \mathrm{cm}^{2}\right)$ was determined. Finally, pre-calculated density of individual polymers were used to determine the thickness. The densities of the hybrids (Hybrid 10, Hybrid 36 and Hybrid 53) were calculated by the taking in to account the wgt $\%$ of the grafted starch and that of the urethanelinked polycaprolactone moiety serving as the backbone. Since the majority component of the backbone polymer was polycaprolactone, the density of polycaprolactone diol $(1.07 \mathrm{~g} / \mathrm{mL})$ was used for the calculation. In terms of the starch fraction, a value of $0.67 \mathrm{~g} / \mathrm{mL}$ was used based on the known density of corn starch. Using these approximations and knowing the wgt \% composition of the various polymers, the densities of Hybrid 10, Hybrid 36 and Hybrid 53 were calculated to be $1.01 \mathrm{~g} / \mathrm{mL}, 0.88 \mathrm{~g} / \mathrm{mL}$ and $0.81 \mathrm{~g} / \mathrm{mL}$, respectively. The density of the base polymer (ULPE 133) was taken to be similar to that of polycaprolactone diol.

The estimated thickness of the Hybrid 36 and Hybrid 53 were in the range of $50-60 \mathrm{~nm}$ whereas the thickness of Hybrid 10 was $15 \mathrm{~nm}$. The thickness of 
spin-coated ULPE-N3-133 was estimated to be $30 \mathrm{~nm}$. We hypothesize that the smaller film thickness observed for Hybrid 10 was most likely due to lower solubility of the Hybrid 10 in the spin coating solvent (HFIP).

2.5 Atomic Force Microscopy (AFM) and Contact Angle Measurements of Thin Films. The surface roughness (root mean square roughness, $\mathrm{R}_{\mathrm{q}}$ ) of the hybrid coatings and the base polymer on QCM gold sensors were determined by tapping mode AFM using a Veeco scanning probe microscope. The scanning rate maintained throughout all the experiments was $1.97 \mathrm{~Hz}$ using an etched single crystal silicon probe. The 3D height traces generated from different hybrids (Hybrid 10, Hybrid 36 and Hybrid 53) and base polymer (ULPE-N3-133) are shown in Figure 2. Measurements were taken on multiple areas of individual coated gold sensors and the data averaged to get the roughness values. Only one chip was used per sample.

Contact angle measurements were performed on a spin coated QCM sensor using a CAM micro contact angle meter. The contact angles were determined by averaging three measurements taken at different locations on the same QCM sensor.

\section{RESULTS AND DISCUSSION}

3.1 Synthesis and Characterization. Generic structures of the hybrid polymers relevant to this study are shown in Scheme 1 and include: three starchcontaining amphiphilic hybrid copolymers, 1 maltodextrin-containing hybrid 
copolymer, and 2 PEG-containing copolymers. Each composition was synthesized in good to excellent yield (46-80\%) using our previously reported graft-to synthetic approach which involves conjugating an alkyne functionalized starch/PEG/maltodextrin to an azide-functionalized ULPE-N3 backbones by copper (I) catalyzed azide alkyne cycloaddition (CuAAC) at $40^{\circ} \mathrm{C}$ [29].

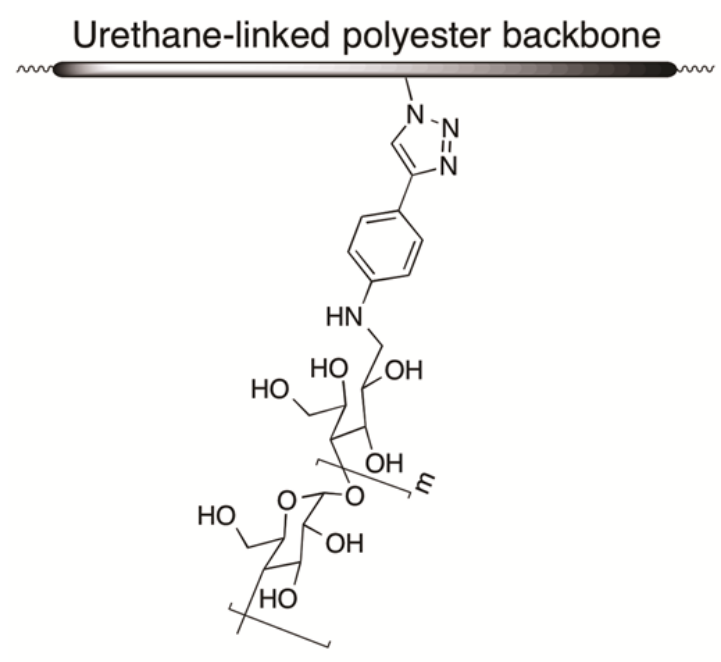

Hybrid 10, 36, 53

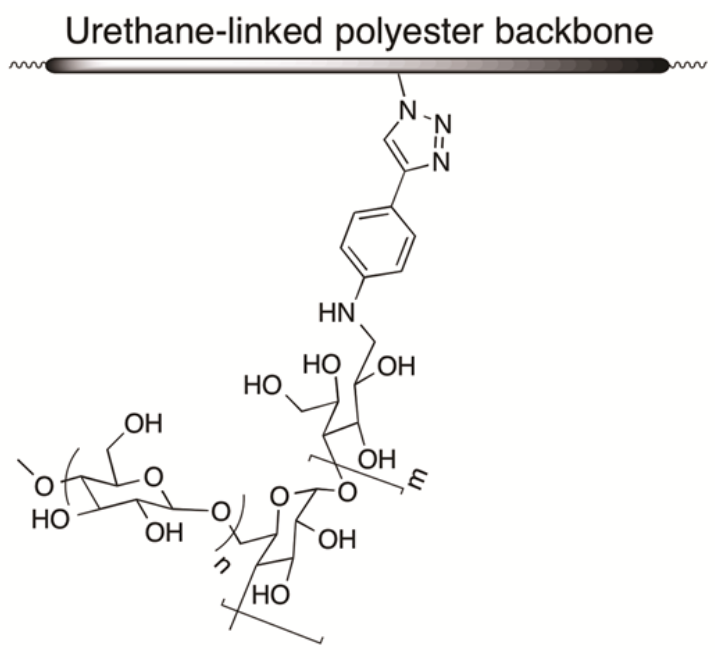

Hybrid MD 24

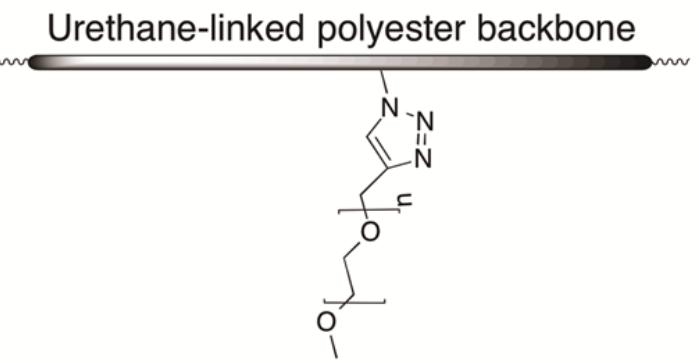

\section{Hybrid PEG 11, 24}

Scheme 1. Structures of urethane-linked polyester amphiphilic graft copolymers that feature three different hydrophilic side chain moieties: high amylose starch, maltodextrin, and PEG. 
Two ULPE-N3 backbone polymers (ULPE-N3-133 and ULPE-N3-182), with different azide loadings, gave rise to three high amylose starch compositions (Hybrid 10, Hybrid 36, Hybrid 53) and one maltodextrin-grafted hybrid polymer (Hybrid MD 24). A correlation diagram between the parent ULPE-N3 and its offspring graft copolymers can be found in the Supporting Information and these relationships are also noted in Table 1. 
Table 1. Summary of key characterization data and relationships between graft copolymers and their corresponding parental base polymer (shown in parentheses). Mass of adsorbed protein was calculated from QCM experiments while graft density and polymer composition was calculated from ${ }^{1} \mathrm{H}$ NMR data.

\begin{tabular}{|c|c|c|c|c|c|}
\hline Entry & $\begin{array}{c}\text { Graft } \\
\text { Density }\end{array}$ & $\begin{array}{c}\text { Wgt \% of } \\
\text { Starch/MD/ } \\
\text { PEG }\end{array}$ & $\begin{array}{c}\text { BSA } \\
\text { Adsorption } \\
\left(\mathrm{ng} / \mathrm{cm}^{2}\right) \\
\text { Mean } \pm \text { SD }\end{array}$ & $\begin{array}{c}\text { Fibrinogen } \\
\text { Adsorption } \\
\left(\mathrm{ng} / \mathrm{cm}^{2}\right) \\
\text { Mean } \pm \text { SD }\end{array}$ & $\begin{array}{c}\text { Surface } \\
\text { Roughness } \\
\text { (nm) }\end{array}$ \\
\hline Bare gold & - & - & $787 \pm 10$ & $1743 \pm 64$ & - \\
\hline $\begin{array}{c}\text { Hybrid } 10 \\
\text { (from ULPE-N3- } \\
\text { 133) }\end{array}$ & $17 \%$ & 10 & $360 \pm 47$ & $2512 \pm 167$ & 6.2 \\
\hline $\begin{array}{c}\text { Hybrid } 36 \\
\text { (from ULPE-N3- } \\
\text { 133) }\end{array}$ & $57 \%$ & 36 & $149 \pm 16$ & $2168 \pm 69$ & 15.4 \\
\hline $\begin{array}{c}\text { Hybrid } 53 \\
\text { (from ULPE-N3- } \\
\text { 182) }\end{array}$ & $75 \%$ & 53 & $60 \pm 15$ & $1514 \pm 185$ & 14.8 \\
\hline $\begin{array}{c}\text { Hybrid MD } 24 \\
\text { (from ULPE-N3- } \\
\text { 133) }\end{array}$ & $43 \%$ & 24 & $247 \pm 42$ & - & - \\
\hline $\begin{array}{c}\text { Hybrid PEG } 11 \\
\text { (from ULPE-N3- } \\
\text { 133) }\end{array}$ & $33 \%$ & 11 & $258 \pm 34$ & - & - \\
\hline $\begin{array}{c}\text { Hybrid PEG } 24 \\
\text { (from ULPE-N3- } \\
\text { 182) }\end{array}$ & $59 \%$ & 24 & $321 \pm 47$ & - & - \\
\hline ULPE-N3-133 & 0 & 0 & $335 \pm 33$ & $2922 \pm 130$ & 5.9 \\
\hline ULPE-N3-182 & 0 & 0 & $394 \pm 17$ & $3069 \pm 96$ & - \\
\hline
\end{tabular}


Of particular note, Hybrid 10 was synthesized by targeting a substoichiometric number of azide reactive sites with starch alkyne units. In this way, a "lightly grafted" copolymer composition was synthesized that contains a total starch content of $10 \mathrm{wgt} \%$. Also important to note is the numbering scheme used throughout this paper. The numerical value in the name corresponds to either an azide loading for the ULPE's (reported in umol/g polymer) or a starch weight percent in the case of the hybrid copolymers. For example, ULPE-N3-133 contains $133 \mathrm{umol} / \mathrm{g}$ of azide density and Hybrid 10 contains $10 \mathrm{wgt} \%$ starch. In addition to the starch-containing copolymers, we also grafted PEG chains onto the ULPE-N3 backbone polymers (Hybrid PEG 11 and Hybrid PEG 24) using the same CuAAC methodology.

We noted small, yet interesting, differences in the CuAAC click reaction for the predominantly linear high amylose starch compared to the alkyne endfunctionalized maltodextrin. At the same reaction temperature $\left(40^{\circ} \mathrm{C}\right)$ and with the same azide-containing ULPE-N3 backbone (in both cases alkyne was used in excess), we achieved low grafting density (27\%) with the maltodextrin macromolecular reagent as compared to the high amylose starch. We hypothesize that branching near the maltodextrin's alkyne end group may hinder access and therefore retard the cycloaddition reaction. Increasing the temperature from $40^{\circ} \mathrm{C}$ to $60^{\circ} \mathrm{C}$ improved the grafting efficiency from 27 to $43 \%$. Figure 1 shows the ${ }^{1} \mathrm{H}$ NMR spectrum of Hybrid MD 24. All of the key resonances are observed that indicate the polysaccharide has been successfully grafted. In particular, both the end group aromatic ring protons and the triazole 
protons are evident and can be compared, quantitatively, to both the anomeric protons and the resonances associated with the hydrophobic backbone.

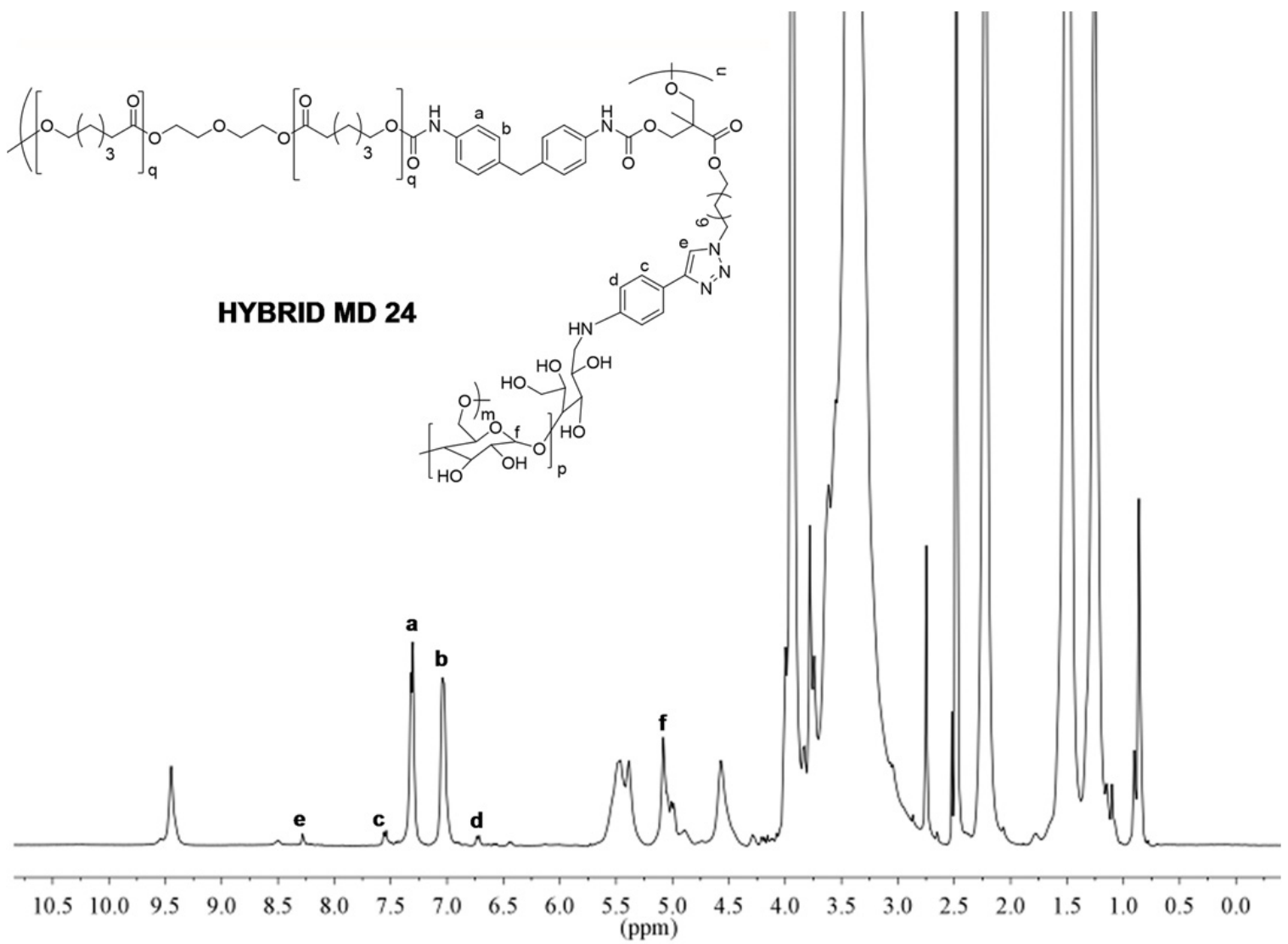

Figure 1. ${ }^{1} \mathrm{H}$ NMR spectrum of Hybrid MD 24 showing key peak assignments associated with the urethane-linked backbone $(a, b)$, triazole linkage $(e)$, aromatic end group $(\mathrm{c}, \mathrm{d})$, and the polysaccharide's anomeric protons (f).

In order to understand the orientation of our polymers films on gold surfaces we investigated the water contact angles of both the ULPE-N3 backbone and the starch-containing hybrid polymers. The predominantly hydrophobic ULPE-N3-133 backbone polymer had a contact angle of $78^{\circ}\left( \pm 1.7^{\circ}\right)$ whereas the two hybrid graft copolymers derived from this particular base polymer (Hybrid 10 and 36 ) had contact angles between $62-65^{\circ}\left( \pm 0.6^{\circ}\right)$. The reduction in contact angle shows an increase in the hydrophilicity of the surface 
upon introduction of the starch grafts. This was expected. Interestingly, there was little variation in the contact angle as the starch content in the copolymer was increased. For example, going from Hybrid 10 to Hybrid 53, the contact angle remained in the range of $61-62^{\circ}\left( \pm 0.6^{\circ}\right)$. The contact angles measured for the starch hybrids are similar to other reported amphiphilic PEG-polyurethane copolymers [36, 37].

The surface topographies of the backbone ULPE and the hybrid polymers as determined by AFM are shown in Figure 2. The calculated roughness $\left(R_{q}\right)$ for ULPE 133 and Hybrid 10 are $5.87 \mathrm{~nm}$ and $6.22 \mathrm{~nm}$ respectively, whereas, for Hybrid 36 and Hybrid 53 the values are $15.4 \mathrm{~nm}$ and $14.8 \mathrm{~nm}$ respectively.

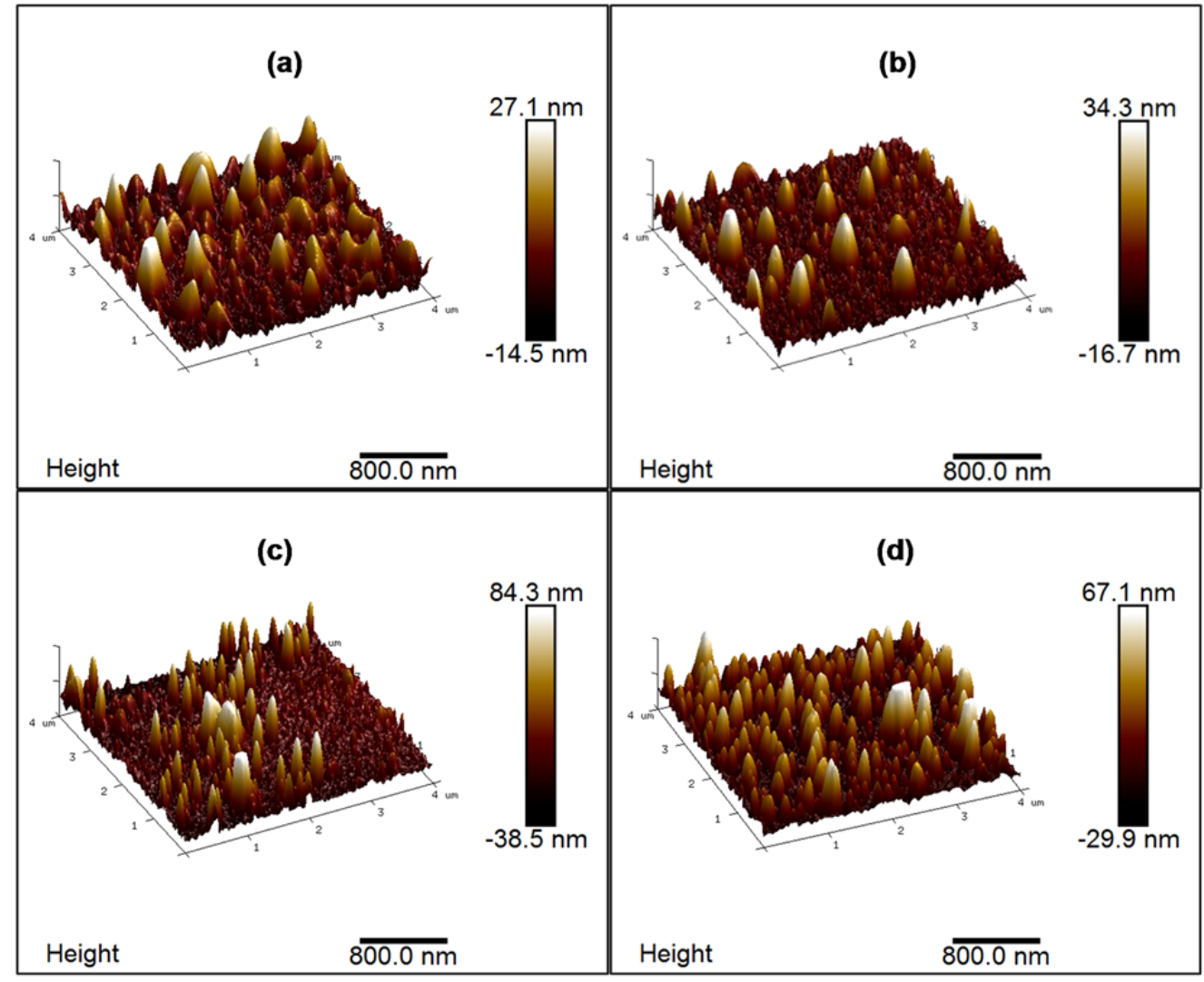

Figure 2. AFM images showing 3D height traces of gold sensor surfaces coated with: (a) ULPE 133 (b) Hybrid 10 (c) Hybrid 36 and (d) Hybrid 53. 
3.2 Protein Adsorption in Starch-Containing Amphiphilic Graft Copolymers as

Studied by QCM. Figure 3 shows the unprocessed time-dependent QCM data ( $3^{\text {rd }}$ overtone) for Hybrids 10,36 , and 53 as well as ULPE-N3-'s 133 and 182 interacting with BSA and fibrinogen.
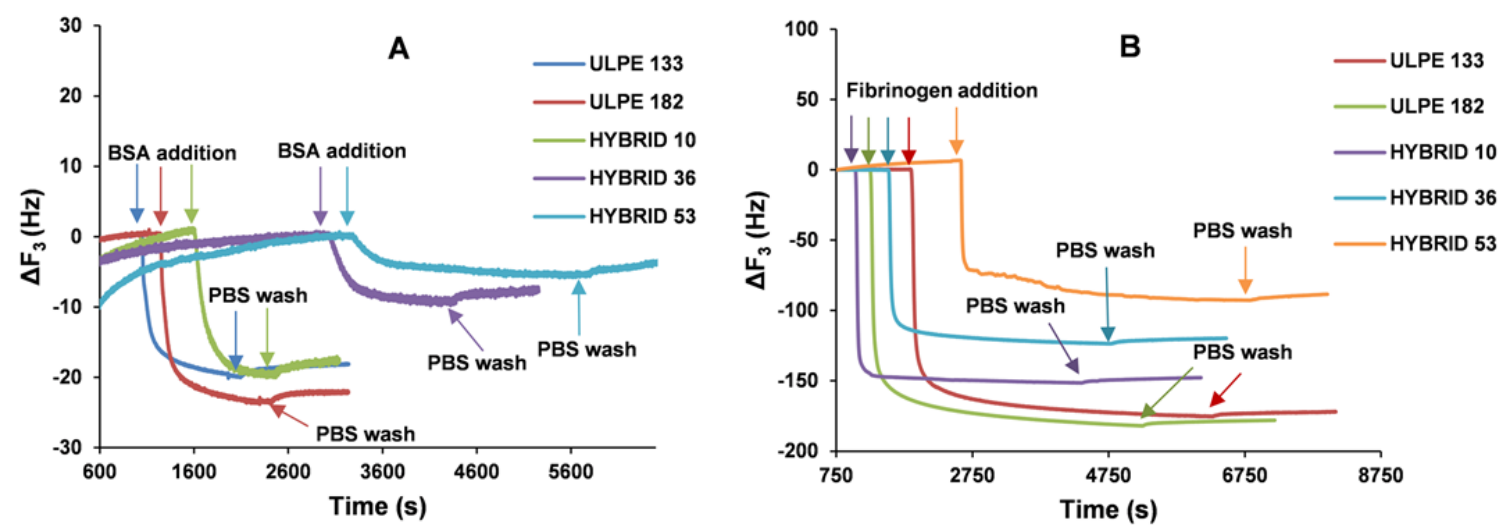

Figure 3. Real-time $Q C M$ frequency changes $(\Delta F)$ for the adsorption of $B S A$ (panel $A$, left) and fibrinogen (panel B, right) on starch-based hybrids (Hybrid 10, 36, 53) and the urethane-linked polyester controls (ULPE-N3-133, ULPE-N3-182).

At the start of the data acquisitions shown in Figure 3, the polymer-coated sensors have already been equilibrated with PBS buffer. All samples experience an initial large drop in frequency (gain in mass) corresponding to the adsorption of BSA or fibrinogen to the hydrated film. After a short equilibration period (yet still under flow conditions), the protein solution is replaced by buffer; at this point a relatively small increase in frequency (mass loss) is observed. We, and others [38] hypothesize that the buffer wash removes loosely bound protein and the polymer surface then re-equilibrates. 
Hybrid 10 and 36 were synthesized by grafting high amylose starch to backbone ULPE-N3-133. Comparing the parent hydrophobic ULPE-N3-133 to amphiphilic Hybrid 10 shows no statistically significant difference in BSA adsorption (see Figure 4) [39]. It seems that low graft densities, as in Hybrid 10, are insufficient to completely shield the polymer surface from available BSA. In the fibrinogen data series, on the other hand, the protein adsorption was lower than its non-polysaccharide control (ULPE-N3-133). Increasing the starch content to 36 wgt \% (i.e. Hybrid 36), however, reduces the quantity of adsorbed BSA by $55 \%$ compared with its base polymer ULPE-N3-133 (Figure 4).
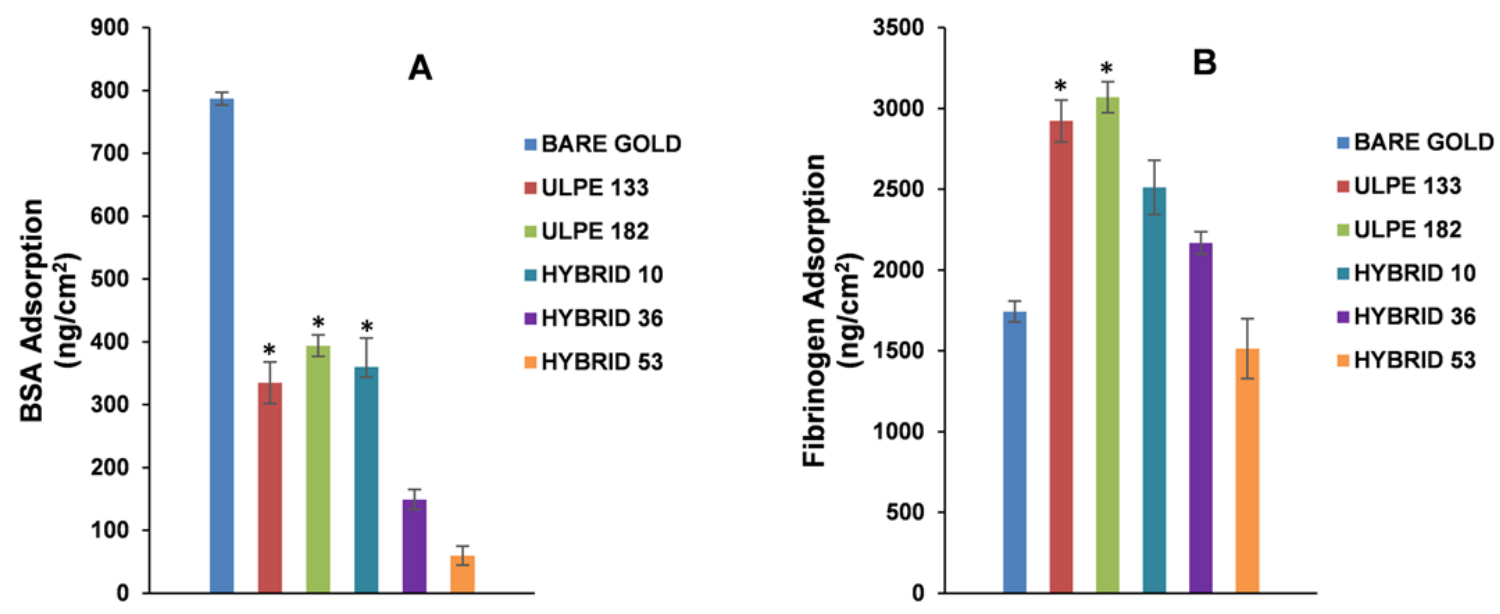

Figure 4. BSA (panel A, left) or fibrinogen (panel B, right) protein adsorption profiles (mass of protein adsorbed $/ \mathrm{cm}^{2}$ ) associated with: bare gold-coated quartz sensor, ULPEN3 backbone polymers, and starch-containing hybrid polymers. A "*" Indicates a lack of statistical significance with the neighboring column(s).

Hybrid 53 was derived from base polymer ULPE-N3-182 and contains the highest quantity of starch in the entire set of amphiphilic graft copolymers. Comparing Hybrid 53 to its parent ULPE-N3-182, we see an $85 \%$ reduction in 
BSA adsorption and a $51 \%$ reduction in fibrinogen adsorption (see Figure 4). Hybrid 53, like the other compositions, is insoluble in water and most organic solvents yet retains excellent film forming properties and thermal stability [29].

In order to study the impact of polysaccharide branching on protein adsorption, we synthesized a maltodextrin graft copolymer by the same methodology. The maltodextrin used in this study is derived from rice starch-a raw material known to be rich in amylopectin (>70\% amylopectin) [40]. The maltodextrin has a dextrose equivalent (DE) of 4-7 giving a $M_{n}$ range of approximately $4200-7300 \mathrm{~g} / \mathrm{mol}$. Hybrid MD 24 showed a $26 \%$ reduction in BSA adsorption compared with its parent ULPE-N3-133 indicating that, even at low grafting density, the polysaccharide impedes protein adsorption. Comparing Hybrid MD 24 to Hybrid PEG 11, both synthesized from ULPE-N3-133 and both having similar graft densities, did not show any substantial differences in protein adsorption. It should be noted that each individual maltodextrin graft has a larger molecular weight than each MPEG graft; so, for the same graft density and lower molecular weight mPEG exerted the same effect on protein repellency as the maltodextrin. Unfortunately, making direct comparisons to the linear starch (e.g. Hybrid 10 or 36 ) was not trivial due to the different overall starch content and, probably more importantly, substantial differences in graft density.

Comparing the protein adsorption inside the three starch-containing graft copolymers shows a clear trend of increasing starch content correlating with decreasing protein adsorption (Figures 3 and 4). The trends observed for BSA are mirrored in the fibrinogen data except that the magnitude of protein adsorbed 
per unit area is substantially larger and the protein repellency conferred on the hydrophobic base polymers is slightly lower. The differences in the magnitude of protein adsorption can be explained by the fact that fibrinogen's molecular weight is $5 x$ that of BSA, therefore, given the same area, one would expect a larger mass change for fibrinogen as compared to BSA. Importantly, though, moving from Hybrid 10 to Hybrid 53, one observes an $83 \%$ reduction in protein adsorption for the BSA experiments whereas fibrinogen is reduced by $40 \%$ in the same series. Overall, the hybrid polymer coatings reduce protein adsorption as compared to a coating of ULPE-N3 or just the bare sensor.

\subsection{Starch-Containing Hybrid Graft Copolymers Compared to PEG Graft Copolymers.}

Two PEG-containing graft copolymers, Hybrid PEG 11 and Hybrid PEG 24, were synthesized from ULPE-N3-133 and ULPE-N3-182, respectively. The $M_{n}$ of the methoxy-terminated PEG grafts was $2 \mathrm{~K}$ across all compositions. BSA adsorption in Hybrid PEG 11, as compared to its control hydrophobic backbone polymer ULPE-N3-133, was reduced by 23\%. Hybrid PEG 24 imparted a 19\% reduction of BSA adsorption compared to its control ULPE-N3-182. Interestingly, Hybrid PEG 24 has a higher PEG content versus Hybrid PEG 11 yet showed a slightly higher protein adsorption. Cheng et.al reported similar behavior in their study with benzyloxy PEG-g-PU systems [37]. The authors postulated that at low graft density, the benzyloxy-terminated PEG chains have ample space to adopt a loop conformation thereby exposing the more hydrophilic PEG backbone to the interface instead of the hydrophobic end groups. As a result of this 
conformational flexibility, protein adsorption was lower compared to a composition with higher PEG content. As the surface density was increased by grafting both short and long chain PEGs to the polyurethane backbone, the benzyloxy terminal groups were forced into more of an extended conformation; the more rigid brush structure resulted in greater hydrophobicity and increased protein adsorption despite having higher PEG content. In our case, as the graft density of the mPEG increased from 33\% (Hybrid PEG 11) to 59\% (Hybrid PEG 24), BSA adsorption increased by $24 \%$. One possible explanation for this data builds on the previous discussion where the increased graft density forces the methoxyPEG chains into a more rigid conformation and therefore presents a more hydrophobic surface to the incoming protein relative to the more sparsely populated surface. It should be pointed out, however, that one major difference between these two studies is the size of the hydrophobic terminal group: benzyloxy versus methoxy.

In order to compare the mPEG-grafted materials to the starch-grafted materials it is important to choose a set of copolymers with similar compositions and grafting densities as each parameter is expected to impact the protein adsorption in different ways. With these parameters in mind, it is instructive to compare starch-containing Hybrid 36 with PEG-containing Hybrid PEG 24. These two polymers have similar graft densities ( $57 \%$ and $59 \%$, respectively) and originate from the same parent ULPE-N3 backbone. We recognize, however, that the two grafting agents, mPEG and linear high amylose starch, have different molecular weights and this may also influence protein adsorption. The 
QCM data with BSA shows that Hybrid 36 adsorbs $54 \%$ less protein relative to Hybrid PEG 24. Although the differences in composition, graft density, and architecture temper our conclusions, we do observe competitive BSA protein resistance in the starch-containing hybrid polymers as compared to the PEGcontaining materials.

\section{CONCLUSIONS}

In summary, we have synthesized and characterized a suite of amphiphilic graft copolymers with the goal to understand how the structure and density of the hydrophilic side chain impacts protein adsorption. QCM was used to measure the real time adsorption of proteins to polymer-coated surfaces thus providing comparative data to evaluate effectiveness. As the high amylose starch content in these hybrid graft copolymers increased, protein adsorption decreased (for both BSA and fibrinogen). Additionally, the polysaccharide pendant chains conferred significant protein resistance to the hydrophobic urethane-linked polyester backbone polymers. For example, BSA adsorption was reduced $85 \%$ in a composition containing 53 wgt \% starch relative to its corresponding backbone polymer alone. Not unexpectedly, branched maltodextrin grafts, even at low graft density, impart protein resistance relative to polymers lacking these hydrophilic side chains. Finally, comparing a similarly constructed high amylosebased hybrid polymer and a mPEG-grafted copolymer we found enhanced protein resistance in the polysaccharide-containing material. These findings may be of significance in the biomaterial design space considering the low cost, 
abundance, and ability to further enhance the functionality (e.g. dye labeling or active agent attachment) of the polysaccharide component as compared to the more conventional PEG-based materials.

\section{ACKNOWLEDGEMENTS}

The authors acknowledge Carmine P. lovine for helpful discussions. Financial support was also made possible by the National Science Foundation under Grant No. CHE 1305117. Lastly, the authors also acknowledge the Dreyfus Foundation for financial support. 


\section{REFERENCES}

1. S. Martwiset, A. E. Koh, W. Chen, Nonfouling characteristics of dextran containing surfaces, Langmuir 22 (2006) 8192-8196.

2. M. Morra, C. Cassineli, Non-fouling properties of polysaccharide-coated surfaces, J. Biomaterial. Sci. Poly. Ed. 10 (1999) 1107-1124.

3. J. Bozja, J. Sherrill, S. Michielsen, I. Stojiljkovic, Porphyrin-based light activated antimicrobial materials, Journal of Polymer Science, Part A: Polymer Chemistry 41 (2003) 2297-2303.

4. A. Hucknall, S. Rangarajan, A. Chilkoti, In pursuit of zero: polymer brushes that resist the adsorption of proteins, Adv. Mater. 21 (2009) 2441-2446.

5. I. Banerjee, R. C. Pangule, R. S. Kane, Antifouling coatings, Adv. Mater. 23 (2011) 690-718.

6. K. L. Prime, G. M. Whitesides, Self-assembled organic monolayers: Model systems for studying adsorption of proteins at surfaces, Science 252 (1991) 1164-1167.

7. K. L. Prime, G. M. Whitesides, Adsorption of proteins onto surfaces containing end-attached oligo(ethylene oxide): a model system using self-assembled monolayers, J. Am. Chem. Soc. 115 (1993) 10714-10721.

8. N. Xia, C. J. May, S. L. McArthur, D. G. Castner, Time-of-flight secondary ion mass spectrometry analysis of conformational changes in adsorbed protein films, Langmuir 18 (2002) 4090-4097.

9. E. Ostuni, R. G. Chapman, R. E. Holmlin, S. Takayama, G. W. Whitesides, Surveying for surfaces that resist the adsorption of proteins, Langmuir 17 (2001) 5605-5620.

10. A. Bodin, M. Linnerborg, J. L. G. Nilsson, A. T. Karlberg, Structure elucidation, synthesis, and contact allergenic activity of a major hydroperoxide formed at autoxidation of the ethoxylated surfactant $\mathrm{C}_{12} \mathrm{E}_{5}$, Chem. Res. Toxicol. 16 (2003) 575-582.

11. J. G. Ok, J. Y. Lee, H. W. Baac, S. H. Tawfick, L. J. Guo, A. J. Hart, Zwitterbased stainless steel with well-defined polysulfobetaine brushes for general bioadhesive control, Materials \& Interfaces 6 (2014) 861-873.

12. O. A. Mkhatresh, F. Heatley, A ${ }^{13} \mathrm{C}$ NMR study of the products and mechanism of the thermal oxidative degradation of poly(ethylene oxide), Macromol. Chem. Phys. 203 (2002) 2273-2280. 
13. L. Reich, S. S. Stivala, Autoxidation of poly(alkylene glycols) in solution, Journal of Applied Polymer Science 13 (1969) 977-988.

14. R. Konradi, B. Pidhatika, A. Mühlebach, M. Textor, Poly-2-methyl-2oxazoline: A peptide-like polymer for protein-repellent surfaces, Langmuir 24 (2008) 613-616.

15. M. Tanaka, A. Mochizuki, T. Shiroya, T. Motomura, K. Shimura, M. Onishi, Y. Okahata, Study on kinetics of early stage protein adsorption on poly(2methoxyethylacrylate) (PMEA) surface, Colloids and Surface A. 203 (2002) 195204.

16. W. Feng, J. L. Brash, S. Zhu, Non-biofouling materials prepared by atom transfer radical polymerization grafting of 2-methacryloloxyethyl phosphorylcholine: Separate effects of graft density and chain length on protein repulsion, Biomaterials 27 (2006) 847-855.

17. Z. Zhang, T. Chao, S. Chen, S. Jiang, Superlow fouling sulfobetaine and carboxybetaine polymers on glass slides, Langmuir 22 (2006) 10072-10077.

18. N. B. Holland, Y. Qiu, M. Ruegsegger, R. E. Marchant, Biomimetic engineering of non-adhesive glycocalyx-like surfaces using oligosaccharide surfactant polymers, Nature 392 (1998) 799-801.

19. Y.Y. Luk, M. Kato, M. Mrksich, Self-assembled monolayers of alkanethiolates presenting mannitol groups are inert to protein adsorption and cell attachment, Langmuir 16 (2000) 9604-9608.

20. M. Hederos, P. Konradsson, B. Liedberg, Synthesis and self-assembly of galactose-terminated alkanethiols and their ability to resist proteins, Langmuir 21 (2005) 2971-2980.

21. R. J. Mancini, J. Lee, H. D. Maynard, Trehalose glycopolymers for stabilization of protein conjugates to environmental stressors, J. Am Chem. Soc. 134 (2012) 8474-8479.

22. D. Kozak, A. Chen, J. Bax, M. Trau, Protein resistance of dextran and dextran-poly(ethylene glycol) copolymer Films, Biofouling 27 (2011) 497-503.

23. J. Dubois, C. Gaudreault, P. Vermette, Biofouling of dextran-derivative layers investigated by quartz crystal microbalance, Colloids and Surfaces B: Biointerface 71 (2009) 293-299.

24. T. M. Allen, The use of glycolipids and hydrophilic polymers in rapid uptake of liposomes by the mononuclear phagocyte system, Adv. Drug Del. Rev. 13 (1994) 285-309. 
25. M. Ombelli, L. Costello, C. Postle, V. Anantharaman, Q. C. Meng, R. J. Composto, D. M. Eckmann, Competitive protein adsorption on polysaccharide and hyaluronate modified surfaces, Biofouling 27 (2011) 505-518.

26. D. Bikiaris, J. Prinos, K. Koutsopoulos, N. Vouroutzis, E. Pavlidou, N. Frangis, C. Panayiotou, LDPE/Plasticized starch blends containing PE-g-MA copolymer as compatibilizer, Polymer Degradation and Stability 59 (1998) 287-291.

27. J. P. Wang, S. J. Yuan, Y. Wang, H. Q. Yu, Synthesis, characterization and application of a novel starch-based flocculant with high flocculation and dewatering properties, Water. Res. 47 (2013) 2643-2648.

28. H. Namazi, M. Mosadegh, A. Dadkhah, New intercalated layer silicate nanocomposites based on synthesized starch-g-PCL prepared via solution intercalation and in situ polymerization methods: A comparative study, Carbohydrate Polymers 75 (2009) 665-669.

29. L. M. Ryno, C. Reese, M. Tolan, J. O'Brien, G. Short, G. Sorriano, J. Nettleton, K. Fulton, P. M. lovine, Amphiphilic graft copolymers from endfunctionalized starches: Synthesis, characterization, thin film preparation, and small molecule loading, Biomacromolecules 15 (2014) 2944-2951.

30. Z. Cao, T. Tsoufis, T. Svaldo-Lanero, A.-S. Duwez, P. Rudolf, K. Loos, The dynamics of complex formation between amylose brushes on gold and fatty acids by QCM-D, Biomacromolecules 14 (2013) 3713-3722.

31. A. Joy, D.M. Cohen, A. Luk, E. Anim-Danso, C. Chen, J. Kohn, Control of surface chemistry, substrate stiffness and cell function in a novel terpolymer methacrylate library, Langmuir 27 (2011) 1891-1899.

32. J. Malmström, H. Agheli, P. Kingshott, D. S. Sutherland, Viscoelastic modeling of highly hydrated laminin layers at homogeneous and nanostructured surfaces: Quantification of protein layer properties using QCM-D and SPR, Langmuir 23 (2007) 9760-9768.

33. O. Altintas, U. Tunca, C. Barner-Kowollik, Star and miktoarm star block (co)polymers via self-assembly of ATRP generated polymer segments featuring hamilton wedge and cyanuric acid binding motifs, Polym. Chem. 2 (2011) 1146-1155.

34. I. Reviakine, D. Johannsmann, R. P. Richter, Hearing what you cannot see and visualizing what you hear: Interpreting quartz crystal microbalance data from solvated interfaces, Anal. Chem. 83 (2011) 8838-8848. 
35. L. Fu, X. Chen, J. He, C. Xiong, H. Ma, Study viscoelasticity of ultrathin poly(oligo(ethylene glycol) methacrylate) brushes by a quartz crystal microbalance with dissipation, Langmuir 24 (2008) 6100-6106.

36. M. F. Shih, M. D. Shau, C. C. Hsieh, J. Y. Cherng, Synthesis and evaluation of poly(hexamethylene-urethane) and PEG-poly(hexamethylene-urethane) and their cholesteryl oleyl carbonate composites for human blood biocompatibility, Molecules 16 (2011) 8181-8197.

37. H. Chen, X. Hu, Y. Zhang, D. Li, Z. Wu, T. Zhang, Effect of chain density and conformation on protein adsorption at PEG-grafted polyurethane surfaces, Colloids and Surfaces B: Biointerfaces 61 (2008) 237-243.

38. G. Anand, F. Zhang, R. J. Linhardt, G. Belfort, Protein-associated water and secondary structure effect removal of blood proteins from metallic substrates, Langmuir 27 (2011) 1830-1836.

39. M. J. Santos-Martinez, I. Inkielewicz-Stepniak, C. Medina K. Rahme, D. M. D'Arcy, D. Fox, J. D. Holmes, H. Zhang, M. W. Radomski, The use of quartz crystal microbalance with dissipation (QCM-D) for studying nanoparticle-induced platelet aggregation, Int. J. Nanomedicine. 7 (2012) 243-255.

40. J. BeMiller, R. Whistler, Starch: Chemistry and Technology. Academic Press: Elsevier, $3^{\text {rd }}$ ed. (2009). 


\section{TABLE OF CONTENTS FIGURE}

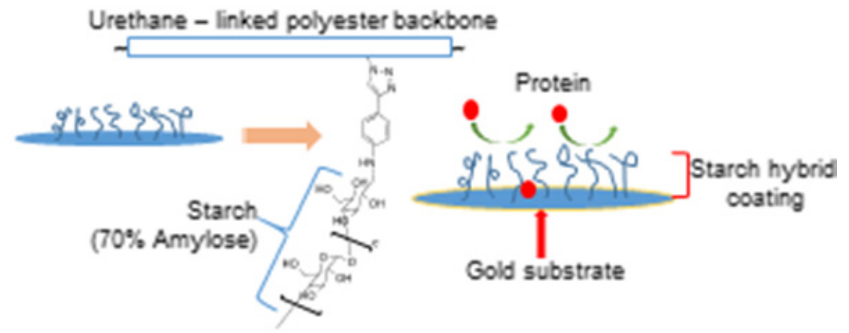




\section{Figure Captions}

Scheme 1. Structures of urethane-linked polyester amphiphilic graft copolymers that feature three different hydrophilic side chain moieties: high amylose starch, maltodextrin, and PEG.

Figure 1. ${ }^{1} \mathrm{H}$ NMR spectrum of Hybrid MD 24 showing key peak assignments associated with the urethane-linked backbone $(a, b)$, triazole linkage $(e)$, aromatic end group (c,d), and the polysaccharide's anomeric protons (f).

Figure 2. AFM images showing 3D height traces of gold sensor surfaces coated with: (a) ULPE 133 (b) Hybrid 10 (c) Hybrid 36 and (d) Hybrid 53.

Figure 3. Real-time $\mathrm{QCM}$ frequency changes $(\triangle \mathrm{F})$ for the adsorption of $\mathrm{BSA}$ (panel A, left) and fibrinogen (panel B, right) on starch-based hybrids (Hybrid 10, $36,53)$ and the urethane-linked polyester controls (ULPE-N3-133, ULPE-N3-182).

Figure 4. BSA (panel $\mathrm{A}$, left) or fibrinogen (panel $\mathrm{B}$, right) protein adsorption profiles (mass of protein adsorbed $/ \mathrm{cm}^{2}$ ) associated with: bare gold-coated quartz sensor, ULPE-N3 backbone polymers, and starch-containing hybrid polymers. A “*” Indicates a lack of statistical significance with the neighboring column(s). 